\title{
A influência do método Pilates na flexibilidade muscular, sintomas e qualidade de vida em mulheres com dismenorreia primária
}

\author{
The influence of the Pilates method on muscular flexibility, symptoms, and \\ quality of life in women with primary dysmenorrhea
}

\author{
Juliany Marques Abreu da Fonseca1 $₫$, Caroline Santos Radmann1, Fabiana Teixeira de Carvalho", \\ Laiana Sepúlveda de Andrade Mesquita ${ }^{1}$
}

${ }^{1}$ Centro de Ciências da Saúde (CCS), Departamento de Fisioterapia, Universidade Estadual do Piauí (UESPI). Teresina, PI.

\section{RESUMO}

Objetivos: Investigar a influência dos exercícios do método Pilates sobre a flexibilidade muscular, sintomatologia e qualidade de vida em mulheres com dismenorreia primária.

Métodos: Foi realizado um ensaio clínico não controlado com 10 universitárias de 18 a 25 anos de idade com dismenorreia primária, que participaram de um protocolo de exercícios baseado no método Pilates. O protocolo constou de 13 exercícios, no solo e com bola, com duração de 50 minutos e frequência de três vezes por semana, totalizando sete semanas. As participantes foram avaliadas antes e após as sete semanas de atendimento pelo teste de distância dedo-solo para flexibilidade muscular, escala visual analógica para dor e o questionário Short Form-36 para qualidade de vida. Os dados foram analisados com o Bioestat $^{\mathbb{E}}$ e apresentados por média e desvio padrão. Após o teste Shapiro-Wilk, aplicou-se teste $t$ de Student ou Wilcoxon, com significância estatística para $p \leq 0,05$.

Resultados: Entre as avaliações feitas antes e depois da aplicação dos exercícios do método Pilates houve aumento da flexibilidade, caracterizado pela redução da distância dedo-solo $(\mathrm{p}=0,007)$. Houve redução da dor, tanto em intensidade $(7,5 \pm 1,6$ para $4,5 \pm 3,2, \mathrm{p}=0,005)$ quanto em duração $(45,6 \pm 19,4$ para $21,6 \pm 16,7$ horas, $p=0,007)$, diminuição percentual dos sintomas mais prevalentes - cansaço $(90 \%$ para $60 \%)$, irritabilidade ( $80 \%$ para $50 \%$ ) e cefaleia (50\% para $20 \%$ ) - e redução do uso de medicação ( $80 \%$ para $40 \%)$. O Short Form-36 mostrou aumento significativo nos escores de qualidade de vida em três dos oito domínios: dor $(45,2 \pm 13,5$ para $59,3 \pm 22,0 ; \mathrm{p}=0,022)$, estado geral de saúde $(68,7 \pm 14,9$ para $80,6 \pm 16,0 ; \mathrm{p}=0,035)$ e saúde mental $(62 \pm 18,5$ para $73,2 \pm 14,3 ; \mathrm{p}=0,038)$.

Conclusões: Além de alívio álgico, os exercícios do método Pilates proporcionaram melhora da flexibilidade muscular, redução da dor e dos desconfortos associados à dismenorreia e influência positiva na qualidade de vida, indicando ser alternativa eficaz para o tratamento sintomático da dismenorreia primária.

DESCRITORES: dismenorreia; qualidade de vida; fisioterapia; terapia por exercício; Pilates.

\section{ABSTRACT}

Aims: To investigate the influence of Pilates on muscular flexibility, symptoms, and quality of life in women with primary dysmenorrhea. Methods: An uncontrolled clinical trial was conducted with 10 university students aged 18 to 25 years with primary dysmenorrhea who participated in an exercise protocol based on the Pilates method. The protocol included thirteen 50-minute floor and ball exercises three times a week for seven weeks. Participants were assessed before and after seven weeks for muscular flexibility by the fingertip-to-floor test, for pain by the visual analog scale, and for quality of life by the Short Form-36 health survey. Data were analyzed with Bioestat ${ }^{\mathbb{E}}$ and presented as mean and standard deviation. After the Shapiro-Wilk test, either Student's t test or Wilcoxon test was applied, and statistical significance was attained when $\mathrm{p} \leq 0.05$.

Results: The assessments before and after the Pilates sessions showed an increase in flexibility, characterized by reduction in the fingertip-tofloor distance $(\mathrm{p}=0.007)$. Pain decreased both in intensity ( $7.5 \pm 1.6$ to $4.5 \pm 3.2, \mathrm{p}=0.005)$ and in length $(45.6 \pm 19.4$ to $21.6 \pm 16.7$ hours $\mathrm{p}=0.007)$; the most prevalent symptoms were relieved - fatigue (from 90 to 60), irritability (from 80 to $50 \%$ ), and headache (from 50 to $20 \%$ ); and medication use was reduced from 80 to $40 \%$. The Short Form-36 showed a significant increase in quality of life scores in three of eight domains: pain $(45.2 \pm 13.5$ to $59.3 \pm 22.0 ; \mathrm{p}=0.022)$, general health $(68.7 \pm 14.9$ to $80.6 \pm 16.0 ; \mathrm{p}=0.035)$, and mental health $(62 \pm 18.5$ to $73.2 \pm 14.3 ; \mathrm{p}=0.038)$ Conclusions: In addition to pain relief, Pilates exercises improved muscular flexibility, reduced pain and discomfort associated with dysmenorrhea, and had a positive influence on quality of life, proving to be an effective alternative for the symptomatic treatment of primary dysmenorrhea.

KEY WORDS: dysmenorrhea; quality of life; physical therapy specialty; exercise therapy; Pilates.

Recebido: fevereiro, 2016

Aceito: abril, 2016

Este artigo está licenciado sob forma de uma licença Creative Common 
Abreviaturas: SF-36, Short Form-36.

\section{INTRODUÇÃO}

A dismenorreia caracteriza-se por menstruação com sintomatologia álgica, que se manifesta por cólica na região pélvica ou abdominal inferior, podendo irradiar-se para a coluna lombossacra e coxas e ser acompanhada por outros sintomas [1,2]. É a queixa ginecológica mais comum em jovens, com prevalência entre $43 \%$ e $93 \%$ da população feminina [3]. Quando ocorre na ausência de causa patológica e apresenta exames normais é classificada como primária [2].

De maneira geral a dismenorreia manifesta-se logo após a menarca, ocorrendo durante os ciclos ovulatórios [4,5], alcançando um pico por volta dos 20 anos de idade [6]. Estudos sugerem relação entre a ocorrência e gravidade álgica e fatores como dieta, sedentarismo, peso, idade, características do ciclo menstrual, hábitos de vida, estresse e ansiedade [3].

A etiopatogenia da dismenorreia envolve aumento dos níveis de prostaglandina, hipercontratilidade uterina, redução do fluxo sanguíneo e consequente isquemia, bem como fatores emocionais associados. O diagnóstico é eminentemente clínico, confirmado pela ausência de patologia pélvica e cuja história inclui as proximidades do início da dismenorreia com a menarca, surgimento de sintomas com o início do fluxo menstrual, duração das cólicas e sua descrição característica [1,3] diferindo de outras entidades clínicas como a síndrome pré-menstrual [7].

De acordo com a intensidade dos sintomas, a dismenorreia pode levar a incapacidade na realização de atividades habituais, comprometendo atividades da vida diária e constituindo causa importante de absenteísmo escolar e laboral; pode ainda ocasionar prejuízos psicossociais e na qualidade de vida. Além disso, leva grande parte da população feminina acometida a tornar-se dependente de medicamentos $[3,5,8]$.

Vários tratamentos são propostos, principalmente com uso de fármacos, os quais geralmente são acompanhados por efeitos adversos e isoladamente podem não ser suficientes. Pesquisas têm evidenciado a eficácia de recursos não farmacológicos, incluindo acupuntura, estimulação elétrica nervosa transcutânea, quiropraxia, osteopatia, banhos de imersão, controle do estresse, medidas alimentares e atividades físicas regulares [8]. A fisioterapia também oferece recursos para diminuir ou eliminar a dor, entre os quais o método
Pilates pode ser uma alternativa eficaz para pacientes com dismenorreia [1,9].

Criado por Joseph Pilates, o método contempla exercícios que dão ênfase ao que ele denominou de power house, centro de força estabilizador do corpo, composto pelos músculos transverso abdominal, multífidos e do assoalho pélvico. A recuperação, a manutenção e a promoção da saúde são obtidas por meio de exercícios que melhoram o condicionamento, a flexibilidade, a postura, a consciência corporal, a coordenação e o controle muscular [10].

O método desenvolvido por Pilates pode ser compreendido em seis princípios básicos: concentração, na qual a atenção na realização do exercício é de suma importância ao aprendizado motor, grande objetivo da técnica; controle, a fim de evitar contrações musculares inadequadas, com vistas a um movimento suave e harmônico; precisão, importante na qualidade do movimento; centramento, referência ao fortalecimento da musculatura do centro de força, que proporciona a estabilização do tronco e o alinhamento biomecânico; respiração, sincronizada à ação muscular; e movimento fluido, de forma controlada e contínua, contribuindo para a manutenção da saúde do corpo $[10,11]$. Além disso, a respiração associada aos exercícios promove melhor oxigenação de cada parte do organismo, [11-13] o que pode interferir positivamente na sintomatologia da dismenorreia. Tal influência é relevante devido ao impacto causado em atividades de vida diária [14] bem como ao risco associado à automedicação adotada pela maioria das mulheres acometidas [15].

Outros estudos já sugeriram exercícios do método Pilates como alternativa não medicamentosa para o alívio álgico, porém não avaliaram o seu impacto sobre a qualidade de vida das pacientes, ou sobre outros fatores associados $[6,16]$. Nesse sentido, buscouse investigar a influência de exercícios do método Pilates sobre a flexibilidade muscular, sintomatologia e qualidade de vida em mulheres com dismenorreia primária.

\section{MÉTODOS}

Foi realizado um ensaio clínico não controlado, com acadêmicas do curso de graduação em fisioterapia de uma universidade pública de Teresina, Piaú, no período de agosto a novembro de 2014. As participantes assinaram o Termo de Consentimento Livre e Esclarecido, conforme a Resolução 466/2012 do Conselho Nacional de Saúde, que rege pesquisas com seres humanos. O projeto foi aprovado pelo Comitê de Ética em Pesquisa da Universidade 
Estadual do Piauí com o parecer número 684.749 de 11/06/2014 e registrado no Clinical Trials com o número NCT02301559.

Um questionário estruturado que teve por base o de Rodrigues et al. [3], aplicado pela pesquisadora, foi respondido por todas as estudantes de fisioterapia do campus que estavam presentes em sala de aula. $\mathrm{O}$ questionário investigava aspectos referentes à dismenorreia, como a regularidade do ciclo menstrual, a proximidade de início dos sintomas com a menarca e a história médica. A dor foi avaliada pela escala visual analógica, que atribui à dor valores de zero a 10 , na qual era assinalada intensidade zero para ausência de dor e 10 ao nível de dor máxima.

Foram incluídas mulheres de 18 a 25 anos, sedentárias, nuligestas, com ciclo menstrual regular, queixa álgica de intensidade igual ou maior a 04 na escala visual analógica, com proximidade entre o início dos sintomas e a menarca, que relataram não ter história de doenças pélvicas diagnosticadas. Foram excluídas aquelas com queixa de dor lombar, uso de dispositivo intrauterino, recusa em participar do estudo e mais de três faltas consecutivas aos atendimentos. Não foi vedado o uso de medicação para alívio álgico durante o estudo, ficando a critério das voluntárias utilizá-la conforme julgassem necessário.

Para caracterização da amostra foram mensurados o peso, a estatura e o índice de massa corpórea conforme parâmetros estabelecidos pela Organização Mundial da Saúde. Para avaliação clínica foi aplicado questionário estruturado, tendo por base o de Rodrigues et al. [3], que investigava características do ciclo menstrual, tratamento e limitações causadas pela dismenorreia.

Analisou-se a flexibilidade muscular pela distância dedo médio-solo, teste no qual mensura-se os centímetros entre a ponta do dedo médio da mão direita e a linha dos pés durante flexão anterior do tronco. Para a avaliação solicitou-se que as participantes realizassem flexão anterior do tronco, com o movimento de tocar os pés com as mãos a partir da posição ortostática sem flexionar os joelhos. Valores além da linha dos pés foram considerados negativos [17].

A qualidade de vida foi verificada pelo questionário Short Form-36 (SF-36), que avalia oito domínios: capacidade funcional, dor, estado geral de saúde, vitalidade, aspectos físicos, sociais, emocionais e saúde mental, apresentando escore final de zero a 100 , onde zero corresponde a pior e 100 a melhor qualidade de vida [18].

Foram realizados atendimentos com aplicação de exercícios do método Pilates, com duração de 50 minutos e frequência de três vezes por semana, totalizando sete semanas de acompanhamento. O protocolo, desenvolvido para a presente pesquisa, constou de 13 exercícios $[13,19]$ no solo e com bola suíça, buscando mobilização pélvica. Os exercícios foram distribuídos gradativamente durante as sessões realizadas no período de acompanhamento, sendo substituídos por movimentos de maior complexidade conforme as participantes evoluíam na realização dos movimentos. O número de repetições foi no máximo 15 (Tabela 1). Os exercícios foram realizados mediante instrução e acompanhamento da pesquisadora, fisioterapeuta com formação no método Pilates. Ocorriam em sala reservada, com as participantes reunidas em pequenos grupos. As participantes foram avaliadas antes e após as sete semanas de atendimento.

Para processamento dos dados utilizou-se o programa Bioestat ${ }^{\circledR}$. Após o teste Shapiro-Wilk para análise da normalidade, aplicou-se teste t de Student para dados normais e teste de Wilcoxon para dados nãonormais. As variáveis foram apresentadas por média e desvio padrão, considerando-se como significância estatística um $\mathrm{p} \leq 0,05$.

Tabela 1. Distribuição dos exercícios do método Pilates no protocolo adaptado para mulheres com dismenorreia primária, ao longo de sete semanas. Teresina, Piauí, 2014

\begin{tabular}{lc}
\hline \multicolumn{1}{c}{ Exercícios } & Repetições \\
\hline Primeira semana & 10 \\
Respiração diafragmática & 10 \\
Balanço da pelve & 10 \\
Elevação da pelve & - \\
Posição básica na bola & 10 \\
Balanço de quadril na bola & 10 \\
Alongamento completo & \\
Segunda e terceira semanas & 3 \\
Enrolar e desenrolar as costas & 15 \\
Balanço da pelve & 15 \\
Elevação da pelve & 15 \\
Balanço de quadril na bola & 10 \\
Exercício de rolar & 15 \\
Alongamento completo & \\
Quarta e quinta semanas & 10 \\
Spine strech forward & 10 \\
Mermaid & 10 \\
Aquec. cintura e quadril & 15 \\
Exercício de rolar & 10 \\
Rolar na bola & 10 \\
Fole & 15 \\
Sexta e sétima semanas & 15 \\
Spine strech forward & 15 \\
Mermaid & 10 \\
Aquecimento cintura e quadril & \\
Rolar na bola & \\
Abdominal com bola & 15 \\
Fole & \\
\hline & \\
\hline
\end{tabular}




\section{RESULTADOS}

O fluxograma de alocação das participantes, elaborado conforme orientações do CONSORT Consolidated Standards of Reporting Trials (www. consort-statement.org/), é apresentado na Figura 1.

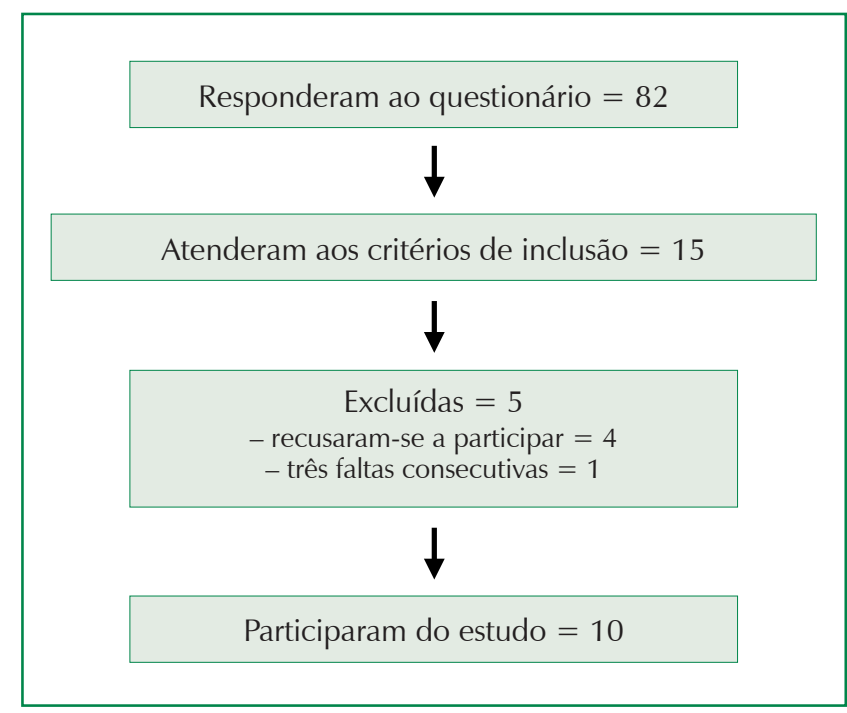

Figura 1. Fluxograma de alocação das participantes. Teresina, Piauí, 2014.

Foram analisadas 10 mulheres com dismenorreia primária, com média de idade 19,2 $\pm 1,39$ anos. Todas eram eutróficas, sendo a média do índice de massa corpórea $20,8 \pm 2,17 \mathrm{~kg} / \mathrm{m}^{2}$. Destas, duas relataram ocorrência da menarca entre oito e 10 anos e oito entre 11 e 15 anos. Absenteísmo em atividades acadêmicas foi declarado por seis participantes. Do grupo, sete procuraram assistência médica para investigação dos sintomas, mas apenas duas relataram utilizar medicação com prescrição médica.

$\mathrm{Na}$ comparação entre a avaliação final, após o protocolo de exercícios do método Pilates, e a realizada antes de iniciar o programa, observou-se redução significativa da média da distância dedo médio-solo, mostrando aumento da flexibilidade muscular. Houve redução significativa na intensidade e no tempo de dor (Tabela 2).

Além da cólica, dentre as queixas mais prevalentes no período menstrual foram relatadas: cansaço, irritabilidade, mastalgia, cefaleia, vômitos, diarreia e desmaio. Destas, cansaço, irritabilidade e cefaleia apresentaram redução após a participação no grupo que recebeu intervenção por meio de exercícios baseados no método Pilates. A necessidade de uso de medicação para alívio álgico, relatada por sete participantes na avaliação inicial, foi mantida por quatro participantes após participação no protocolo (Tabela 3).

O SF-36, questionário pelo qual se avaliou a qualidade de vida das participantes, identificou melhora significativa, após o programa de exercícios, em três dos oito domínios: dor, estado geral de saúde e saúde mental (Tabela 4).

Tabela 2. Flexibilidade muscular, frequência e intensidade de dor antes e após realização dos exercícios do método Pilates por 10 mulheres com dismenorreia primária. Teresina, Piauí, 2014

\begin{tabular}{lccc|} 
& $\begin{array}{c}\text { Antes } \\
\text { Média } \pm \text { DP }\end{array}$ & $\begin{array}{c}\text { Depois } \\
\text { Média } \pm \text { DP }\end{array}$ & p \\
\hline Flexibilidade $(\mathrm{cm})$ & $6,11 \pm 1,7$ & $2,38 \pm 3,8$ & $0,007^{\mathrm{w}}$ \\
Tempo de dor (horas) & $45,6 \pm 19,4$ & $21,6 \pm 16,7$ & $0,007^{\mathrm{w}}$ \\
Intensidade de dor (EVA) & $7,5 \pm 1,6$ & $4,5 \pm 3,2$ & $0,005^{\mathrm{t}}$
\end{tabular}

DP, desvio padrão; EVA, escala visual analógica para verificação da intensidade da dor.

t: teste t de Student; w: teste de Wilcoxon.

Tabela 3. Uso da medicação e sintomas mais prevalentes no período menstrual antes e após realização de protocolo de exercícios do método Pilates por 10 mulheres com dismenorreia primária. Teresina, Piauí, 2014

\begin{tabular}{lll}
\hline & $\begin{array}{c}\text { Antes } \\
\mathbf{n}(\%)\end{array}$ & $\begin{array}{c}\text { Depois } \\
\mathbf{n}(\%)\end{array}$ \\
\hline $\begin{array}{l}\text { Sintomas } \\
\text { Cansaço }\end{array}$ & $9(90)$ & $6(60)$ \\
$\quad$ Irritabilidade & $8(80)$ & $5(50)$ \\
$\quad$ Cefaleia & $5(50)$ & $2(20)$ \\
Uso de medicação antiálgica & $8(80)$ & $4(40)$ \\
\hline
\end{tabular}

Tabela 4. Escores obtidos na avaliação da qualidade de vida pelo questionário SF-36 antes e após realização de protocolo de exercícios do método Pilates por 10 mulheres com dismenorreia primária. Teresina, Piauí, 2014

\begin{tabular}{|lccc|}
\hline \multicolumn{1}{|c}{ Domínios do SF-36 } & $\begin{array}{c}\text { Avaliação } \\
\text { (Média } \pm \\
\text { DP) }\end{array}$ & $\begin{array}{c}\text { Reavaliação } \\
\text { (Média } \pm \\
\text { DP) }\end{array}$ & p \\
\hline Capacidade funcional & $74 \pm 26,8$ & $69,5 \pm 23,5$ & $0,4423^{\mathrm{t}}$ \\
\hline Aspectos físicos & $45 \pm 48,3$ & $60 \pm 39,4$ & $0,4631^{\mathrm{w}}$ \\
\hline Dor & $45,2 \pm 13,5$ & $59,3 \pm 22$ & $\mathbf{0 , 0 2 2 3}^{\mathrm{t}}$ \\
\hline Estado geral de saúde & $68,7 \pm 14,9$ & $80,6 \pm 16$ & $\mathbf{0 , 0 3 5 2}^{\mathrm{t}}$ \\
\hline Vitalidade & $55 \pm 13,9$ & $57,5 \pm 12,7$ & $0,521^{\mathrm{t}}$ \\
\hline Aspectos sociais & $63,7 \pm 22,3$ & $72,4 \pm 19,3$ & $0,1743^{\mathrm{t}}$ \\
\hline Aspectos emocionais & $23,3 \pm 27,4$ & $59,9 \pm 46,6$ & $0,0929^{\mathrm{w}}$ \\
\hline Saúde mental & $62 \pm 18,5$ & $73,2 \pm 14,3$ & $\mathbf{0 , 0 3 8 2}^{\mathrm{w}}$ \\
\hline
\end{tabular}

DP, desvio padrão.

t: teste $\mathrm{t}$ de Student; w: teste de Wilcoxon. 


\section{DISCUSSÃO}

Após o protocolo de exercícios do método Pilates em mulheres com dismenorreia primária houve melhora da flexibilidade muscular, redução da dor, diminuição na ocorrência dos sintomas associados mais prevalentes e redução do uso de medicação. Em relação à qualidade de vida o SF-36 identificou melhora significativa em três dos oito domínios.

A maioria das participantes apresentou idade de menarca entre 11 e 15 anos, intervalo semelhante ao encontrado na literatura $[3,5,15,20,21]$. O absenteísmo em atividades acadêmicas relatado por $60 \%$ das universitárias com dismenorreia é condizente com outros estudos [15,22,23]. Nunes et al. [22] relatam que 30 a 70\% das mulheres recorrem à automedicação no período menstrual; uma proporção superior foi encontrada no presente estudo, $80 \%$. Destas, apenas $20 \%$ utilizavam medicação com prescrição médica, índice semelhante ao apresentado por Yáñez et al. [15] e Sezeremeta et al. [20] e que representa fator alarmante devido aos riscos associados à automedicação.

A melhora da flexibilidade muscular pode ter colaborado para o alívio álgico, uma vez que constitui um importante componente da aptidão física relacionado à saúde. Marchesoni et al. [11] afirmam que a flexibilidade reduzida pode-se associar a problemas posturais, algias, diminuição da vascularização local e aumento de tensões neuromusculares, fatores que podem interferir na dismenorreia. Os exercícios do método Pilates atuam na flexibilidade pelo emprego de alongamentos, mantidos por um determinado tempo [12].

Foi observada redução significativa não apenas da intensidade de dor durante o período menstrual, achado semelhante ao de Araújo et al. [6] e Silva et al. [16], mas também no tempo de dor, fator ainda não abordado por outros estudos e que também incrementa o benefício analgésico obtido. Esse aumento do limiar álgico associado à prática regular de exercício é atribuído à melhor adequação do metabolismo e aporte sanguíneo tecidual, principalmente à região pélvica, como também à maior secreção de neurotransmissores, com ênfase à endorfina [2] que exerce influência no sistema reprodutivo, no ciclo menstrual, na analgesia, nas alterações do humor e em outras funções do organismo [23]. Destaca-se ainda a importância da adaptação dos exercícios às condições físicas das praticantes, uma vez que a complexidade dos exercícios e o número de repetições foram aumentados gradativamente, o que além de favorecer a atividade sem a exacerbação do sintoma álgico, tende a reduzir a taxa de abandono $[24,25]$.
O método Pilates atende à realidade de praticantes de qualquer idade, sexo ou nível de aptidão, pois os exercícios podem ser adaptados conforme as condições físicas [11]. Além disso, trabalha diretamente com os músculos mais profundos do corpo, criando um núcleo forte, sem a dor associada a exercícios convencionais, sempre com ênfase na qualidade do movimento em detrimento da quantidade de repetições $[10,19]$. Por isso enfatizamos o alongamento e a mobilidade da região pélvica sem exigir muitas repetições.

São indicados para o tratamento da dismenorreia exercícios que mobilizem a região pélvica, utilizando movimentos de inclinação e rotação, podendo utilizar também a bola como recurso. Tais movimentos melhoram a coordenação muscular, alongam delicadamente os músculos e promovem o relaxamento dessas estruturas, proporcionando irrigação sanguínea mais abundante nessa região [26]. Além disso a respiração associada aos movimentos, presente nos exercícios do método Pilates, oxigena melhor todo o corpo e proporciona massagem abdominal pelo movimento realizado pelo diafragma [13].

Quanto à frequência dos atendimentos, estudos que abordaram exercícios do método Pilates em mulheres com dismenorreia relatam não haver ainda consenso, mas trazem em sua metodologia dois atendimentos semanais, demonstrando bons resultados $[6,16]$. Entretanto, optou-se por realizar três atendimentos semanais, frequência citada como ideal por Marés et al. [10]. Quanto à duração, o intervalo de 50 minutos foi sugerido como tempo adequado para realização dos exercícios sem levar à exaustão, o que vai de encontro ao que afirma Cunha et al. [23] quando diz que o exercício é o principal estímulo para secreção da betaendorfina. Entretanto, o treinamento excessivo pode diminuir a concentração dessa endorfina, prejudicando os efeitos benéficos do exercício.

Por atuar globalmente, pelo princípio da integração concebido por Siler [19] o qual vê o corpo como um todo e exige uma atividade completa, os exercícios do método Pilates têm potencial para influenciar não só o quadro álgico ocasionado pelas cólicas, mas também alguns sintomas associados. No presente estudo, os sintomas apontados com maior prevalência pelas voluntárias apresentaram redução após a realização dos exercícios, corroborando com os elevados índices encontrados por outros autores [20,27].

Consequentemente à melhora da dor e dos sintomas associados, houve redução na medicação utilizada. O uso de fármacos, não vedado pelo estudo, foi delimitado pela necessidade sentida conforme o quadro álgico. Ao final dos atendimentos, o número 
de mulheres que relatavam uso de medicamentos reduziu-se de oito para quatro o que demonstra o impacto do exercício para analgesia e melhora do quadro geral de mulheres com dismenorreia $[6,16,22]$.

Como o uso de medicamento não foi vedado durante a aplicação do protocolo, essa poderia ser considerada uma limitação do estudo, no sentido de que a melhora dos sintomas em alguns casos poderia não ser creditada apenas aos exercícios realizados, mas também ao uso de medicação. Entretanto, o fato de ter havido diminuição do número de usuárias e da quantidade necessária de medicação, torna essa limitação improvável.

Analisou-se como o protocolo poderia interferir na qualidade de vida por meio do questionário SF-36. Na avaliação inicial foram observados menores índices nos domínios emocional, físico, dor e vitalidade, achados condizentes com Frare et al. [21], que explicam que por ser a dismenorreia uma síndrome dolorosa que primariamente atinge a saúde física, gera pontuações baixas nos quesitos dor e estado geral de saúde. Com a persistência dos sintomas, o domínio vitalidade diminui, rebaixando assim os aspectos emocionais [21].

Na reavaliação pelo SF-36 após a aplicação dos exercícios observou-se melhora significativa para os domínios dor, estado geral de saúde e saúde mental. Aspectos emocionais, sociais, físicos e vitalidade também demonstraram uma tendência à melhora, porém sem significado estatístico. Provavelmente a redução da dor e dos sintomas interferiu positivamente na qualidade de vida, sendo mais evidente quando avaliados aspectos psicológicos, dado o impacto da dor nesse domínio [28]. Ressalta-se ainda que os atendimentos ocorreram em pequenos grupos. Estudos sugerem que a interação com outras pessoas no ambiente de exercício também estaria relacionada às mudanças positivas no humor e aspectos psicológicos, abordando como fatores determinantes o prazer pela atividade, a interação social e a distração [24].

A capacidade funcional foi o único domínio que apresentou uma tendência à diminuição, porém não significativa, conforme os escores obtidos. Essa diminuição poderia ocorrer por causa da melhor percepção corporal, de sintomas e de suas limitações por parte das voluntárias no momento da reavaliação, como comentam Nogueira e Navega [29].

Podem ser citadas como limitações deste estudo a pequena amostra e o tempo reduzido de acompanhamento das participantes. Entretanto, mesmo considerando essas limitações, pode-se concluir, pelos resultados obtidos nessa amostra de mulheres com dismenorreia primária, que o programa de sete semanas com o método Pilates proporcionou melhora da flexibilidade muscular, redução da intensidade e frequência do quadro álgico e dos sintomas associados, assim como influência positiva na qualidade de vida e redução do uso de medicação. Assim, o método Pilates pode ser uma alternativa não farmacológica simples e de baixo custo para o tratamento sintomático da dismenorreia primária.

\section{NOTA}

Declaração de conflitos de interesse

Os autores declaram não haver conflitos de interesse relevantes ao conteúdo deste estudo.

\section{REFERÊNCIAS}

1. Fae A, Pivetta HMF. Efeitos da massagem do tecido conjuntivo e cinesioterapia no tratamento da dismenorréia primária. Fisioter Bras. 2010;11(2):92-8.

2. Quintana LM, Heinz LN, Portes LA, Alfieri FM. Influência do nível de atividade física na dismenorreia. Rev Bras Ativ Fís Saúde. 2010;15(2):101-4.

3. Rodrigues AC, Gala S, Neves A, Pinto C, Meirelles C, Frutuoso C, Vítor ME. Dismenorreia em adolescentes e jovens adultas: prevalência, factores associados e limitações na vida diária. Acta Med Port. 2011;24(S2):383-92.

4. Dawood MY. Primary dysmenorrhea: advances in pathogenesis and management. Obstet Gynecol. 2006 Aug;108(2):428-41. http://dx.doi.org/10.1097/01.AOG.0000230214.26638.0c

5. Brito SA, Marques CC, Alves DS, Alexandre AC. Prevalence of dysmenorrhea in undergraduate health courses at an institution of higher education. Rev Enferm UFPE. 2012;6(6):1386-94. http://dx.doi.org/10.5205/reuol.2365-18138-1-LE.0606201216

6. Araújo LM, Silva JMN, Bastos WT, Ventura PL. Diminuição da dor em mulheres com dismenorreia primária, tratadas pelo método Pilates. Rev Dor. 2012;13(2):119-23. http://dx.doi.org/10.1590/S1806-00132012000200004

7. Teixeira ALS, Oliveira ECM, Dias MRC. Relação entre o nível de atividade física e a incidência da síndrome pré-menstrual. Rev Bras Ginecol Obstet. 2013;35(2):84-9. http://dx.doi.org/10.1590/s0100-72032013000500004 
8. Haidar MA, Dardes RCM, Navarro APCS. Como diagnosticar e tratar dismenorreia. RBM Rev Bras Med. 2012;69(Esp GO 2):14-8.

9. Baldan CS, Freitas CD, Zambello L. Estimulação elétrica nervosa transcutânea (TENS) alivia a dismenorreia primária: estudo clínico, controlado e randomizado. J Health Sci Inst. 2013;31(2):193-6.

10. Marés G, Oliveira KB, Piazza MC, Preis C, Bertassoni Neto L. A importância da estabilização central no método Pilates: uma revisão sistemática. Fisioter Mov. 2012;25(2):445-51. http://dx.doi.org/10.1590/S0103-51502012000200022

11. Marchesoni C, Martins R, Sales R. Método Pilates e aptidão física relacionada à saúde. EFDeportes [Internet]. 2010 [citado 2016];15(150). Disponível em: http://www.efdeportes.com/

12. Comunello JF. Benefícios do método Pilates e sua aplicação na reabilitação. Instituto Salus. 2011 maio-jun;1-12.

13. Rodriguez J. Pilates 101 exercícios passo a passo. São Paulo: Marco Zero; 2009.

14. Ribeiro CP, Hardy E, Hebling EM. Preferências de mulheres brasileiras quanto a mudanças na menstruação. Rev Bras Ginecol Obstet. 2007;29(2):74-9. http://dx.doi.org/10.1590/S0100-72032007000200003

15. Yáñez N, Bautista-Roa SJ, Ruiz-Sternberg JE, Ruiz-Sternberg AM. Prevalencia y factores asociados a dismenorrea en estudiantes de ciencias de la salud. Rev Cienc Salud. 2010;8(3):37-48.

16. Silva MJL, Freitas CD, Civile VT, Nardini AG. Efeito do método Pilates com bola em mulheres com dismenorreia primária. J Health Sci Inst. 2014;32(1):78-81.

17. Abreu EMC, De Paula Junior AR, Oliveira MA. Avaliação da postura e da flexibilidade em adultos jovens antes e após tratamento através do Método de Reeducação Postural Global. Fisioter Bras. 2010;11(3):1. Seção especial.

18. Ciconelli, RM, Ferraz MB, Santos W, Meinao I, Quaresma MR. Tradução para a língua portuguesa e validação do questionário genérico de avaliação de qualidade de vida SF-36 (Brasil SF-36). Rev Bras Reumatol. 1999;39(3):143-50.

19. Siler B. O corpo Pilates: um guia para fortalecimento, alongamento e tonificação sem o uso de máquinas. São Paulo: Sumus Editorial; 2008.

20. Sezeremeta DC, Carvalho MSS, Vrecchi MR, Marafon RGC, Crespilho LC, Pagotto JP, Mortean ECM. Dismenorreia: Ocorrência na vida de acadêmicas da área de saúde. UNOPAR Cient Ciênc Biol Saúde. 2013;15(2):123-6.

21. Frare JC, Tomadon A, Silva JR. Prevalência da dismenorreia e seu efeito na qualidade de vida entre mulheres jovens. Rev Bras Ciênc Saúde. 2014;12(39):15-20.

22. Nunes JMO, Rodrigues JA, Moura MSF, Batista SRC, Coutinho S, Hazime FA, Barbosa, ALR. Prevalência de dismenorreia em universitárias e sua relação com absenteísmo escolar, exercício físico e uso de medicamentos. Rev Bras Promoç Saúde. 2013;26(3):381-6.

23. Cunha GS, Ribeiro JL, Oliveira AR. Níveis de beta-endorfina em resposta ao exercício e no sobretreinamento. Arq Bras Endocrinol Metab. 2008;52(4):589-98. http://dx.doi.org/10.1590/S0004-27302008000400004

24. Gonçalves E, Cosentino RC. Distress: qual a influência do exercício físico neste conceito. Rev Bras Ciênc Saúde. 2007;3(12):50-4.

25. Souza JB. Poderia a atividade física induzir analgesia em pacientes com dor crônica? Rev Bras Med Esporte. 2009;15(2): 145-50. http://dx.doi.org/10.1590/S1517-86922009000200013

26. Ferreira EJ, Azanki NC, Batista AC, Albernaz C. Atuação da fisioterapia na dismenorreia primária. Vita Sanitas. 2010 jandez;(4):57-72.

27. Datrino NB, Grillo DE. Utilização da dança do ventre como minimizador de dores na dismenorreia primária. In: VII Jornada de Iniciação Científica. São Paulo: Universidade Presbiteriana Mackenzie; 2011.

28. Barcelos PR, Conde DM, Deus JM, Martinez EZ. Qualidade de vida de mulheres com dor pélvica crônica: um estudo de corte transversal analítico. Rev Bras Ginecol Obstet. 2010;32(5):247-53.

29. Nogueira HC, Navega MT. Influência da escola de postura na qualidade de vida, capacidade funcional, intensidade de dor e flexibilidade de trabalhadores administrativos. Fisioter Pesqui. 2011;18(4)353-8. http://dx.doi.org/10.1590/S180929502011000400010 C 